\title{
Variational Method for Nuclear Matter with an Explicit Energy Functional
}

\author{
Masatoshi Takano* \\ Research Institute for Science and Engineering, Waseda University \\ Department of Physics and Applied Physics, Waseda University \\ 3-4-1 Okubo, Shinjuku-ku, Tokyo 169-8555, Japan \\ E-mail: takanomewaseda.ip
}

\section{Hajime Togashi}

Department of Physics and Applied Physics, Waseda University

3-4-1 Okubo, Shinjuku-ku, Tokyo 169-8555, Japan

E-mail: hajime togashieruri.waseda.jp

\section{Sachiko Yamamuro}

Department of Physics, Faculty of Science and Technology, Tokyo University of Science Yamazaki 2641, Noda, Chiba 278-8510, Japan.

E-mail: i6211623ded.tus.ac.jp

\section{Ken'ichiro Nakazato}

Department of Physics, Faculty of Science and Technology, Tokyo University of Science Yamazaki 2641, Noda, Chiba 278-8510, Japan.

E-mail: nakazatodrs.tus.ac.jp

\section{Hideyuki Suzuki}

Department of Physics, Faculty of Science and Technology, Tokyo University of Science Yamazaki 2641, Noda, Chiba 278-8510, Japan.

E-mail: suzukihers.tus.ac.jp

We report on the status of two variational studies of the nuclear equation of state (EOS). One is the study of the variational method with an explicit energy functional (EEF). We have been studying the EEF with two-body central and tensor forces, and, in this paper, we extend the EEF to take into account the state-independent three-body force. The calculated energy of neutron matter with the two-body v6' potential and the repulsive part of the UIX potential is close to that with the Monte Carlo method using the full UIX. The maximum mass of the neutron star with this EOS is about $2.5 M_{\odot}$. The other study is a construction of a new nuclear EOS table for supernova numerical simulations that is based on the cluster variational method. Starting from the nuclear Hamiltonian with the AV18 two-body force and the UIX three-body force, the EOS of uniform hot asymmetric nuclear matter is calculated using the method of Schmidt and Pandharipande. For non-uniform nuclear matter, the free energy of a Wigner-Seitz cell is calculated in the ThomasFermi approximation. At zero temperature, a reasonable phase diagram is obtained.

XII International Symposium on Nuclei in the Cosmos

August 5-12, 2012

Cairns, Australia 


\section{Introduction}

The variational many-body theory is a powerful tool for obtaining a reliable nuclear equation of state (EOS) that governs the structure and evolution of neutron stars. We have been studying a new type of variational method for calculating the energies per nucleon, $E / N$, of infinite nuclear matter at zero temperature starting from the realistic nuclear forces [U, []]. In this method, we construct an explicit energy functional (EEF) that expresses $E / N$ as a functional of variational functions. After obtaining an appropriate energy functional, we can derive the Euler-Lagrange equations and calculate the minimized energy by solving them.

In recent years, we refined EEF with the two-body nuclear force consisting of the central and tensor components. Furthermore, we are extending EEF so that it considers the nuclear three-body force (TBF), which plays an important role in the nuclear EOS. In the first part of this paper, we report this extension of the EEF.

We have also been constructing a new nuclear EOS for core-collapse supernova (SN) hydrodynamic simulations based on the cluster variational method, starting from the realistic nuclear forces [B, 团]. Since the SN-EOSs constructed so far have been based on particular nuclear models, the SN-EOS derived directly from nuclear forces is valuable for studies of $\mathrm{SNe}$ and other astrophysical phenomena related to high-density matter. In the latter part of this paper, we report the current status of our project of constructing this SN-EOS.

\section{Explicit Energy Functional for Neutron Matter}

In this section, we propose the following energy expression of infinite neutron matter in which two neutrons interact through the v6' two-body nuclear potential including the central potential $V_{\mathrm{Cs}}(r)$ ( $s$ is the total spin of two neutrons) and the tensor potential $V_{\mathrm{T}}(r)$ :

$$
\begin{aligned}
\frac{E_{2}}{N}= & \frac{3}{5} E_{\mathrm{F}}+2 \pi \rho \int_{0}^{\infty}\left[\sum_{s=0}^{1} F_{s}(r) V_{\mathrm{C} s}(r)+F_{\mathrm{T}}(r) V_{\mathrm{T}}(r)\right] r^{2} d r \\
& +\frac{\pi \hbar^{2} \rho}{2 m} \int_{0}^{\infty} \sum_{s=0}^{1} F_{\mathrm{C} s}(r)\left[\frac{1}{F_{\mathrm{C} s}(r)} \frac{d F_{\mathrm{C} s}(r)}{d r}-\frac{1}{F_{\mathrm{Fs}}(r)} \frac{d F_{\mathrm{F} s}(r)}{d r}\right] r^{2} d r \\
& +\frac{16 \pi \hbar^{2} \rho}{m} \int_{0}^{\infty}\left\{\left[\frac{d g_{\mathrm{T}}(r)}{d r}\right]+\frac{6}{r^{2}}\left[g_{\mathrm{T}}(r)\right]^{2}\right\} F_{\mathrm{F} 1}(r) r^{2} d r \\
& -\frac{\hbar^{2}}{16 \pi m \rho} \int_{0}^{\infty} \sum_{n=1}^{3} w_{n} \frac{\left[S_{\mathrm{c} n}(k)+2 S_{\mathrm{F}}(k)-3\right]\left[S_{\mathrm{c} n}(k)-S_{\mathrm{F}}(k)\right]^{2}}{S_{\mathrm{c} n}(k) / S_{\mathrm{F}}(k)} k^{4} d k+\frac{E_{\mathrm{o}}}{N}
\end{aligned}
$$

On the right-hand side of Eq. (2.1), $E_{\mathrm{F}}$ is the Fermi energy, $\rho$ is the neutron number density, $m$ is the mass of a neutron, and $F_{s}(r)$ and $F_{\mathrm{T}}(r)$ are the radial and tensor distribution functions, defined as

$$
\begin{aligned}
& F_{S}(r)=\Omega^{2} \sum_{\text {spin }} \int \Psi^{\dagger}\left(x_{1}, x_{2}, \ldots, x_{N}\right) P_{s 12} \Psi\left(x_{1}, x_{2}, \ldots, x_{N}\right) d \mathbf{r}_{3} d \mathbf{r}_{4} \ldots d \mathbf{r}_{N}, \\
& F_{\mathrm{T}}(r)=\Omega^{2} \sum_{\text {spin }} \int \Psi^{\dagger}\left(x_{1}, x_{2}, \ldots, x_{N}\right) S_{\mathrm{T} 12} \Psi\left(x_{1}, x_{2}, \ldots, x_{N}\right) d \mathbf{r}_{3} d \mathbf{r}_{4} \ldots d \mathbf{r}_{N} .
\end{aligned}
$$

${ }^{*}$ Speaker. 
Here, $\Omega$ is the volume of the system, $\Psi$ is the wave function, and $P_{s i j}$ and $S_{\mathrm{T} i j}$ are the spin-projection operator and tensor operator operating onto an $(i, j)$ neutron pair, respectively. $F_{\mathrm{Cs}}(r)$ and $g_{\mathrm{T}}(r)$ in Eq. (2.1) are auxiliary functions defined with $F_{s}(r)$ and $F_{\mathrm{T}}(r)$. Explicit definitions of these functions are given in Ref. []. Structure functions $S_{\mathrm{c} n}(k)$ are defined as

$$
\begin{aligned}
& S_{\mathrm{c} 1}(k)=\frac{1}{N}\left\langle\left|\sum_{i=1}^{N} \exp \left[i \mathbf{k} \cdot \mathbf{r}_{i}\right]\right|^{2}\right\rangle=1+S_{1}(k)+S_{0}(k) \geq 0 \\
& S_{\mathrm{c} 2}(k)=\frac{1}{N k^{2}}\left\langle\left|\sum_{i=1}^{N}\left(\sigma_{i} \cdot \mathbf{k}\right) \exp \left[i \mathbf{k} \cdot \mathbf{r}_{i}\right]\right|^{2}\right\rangle=1+\frac{S_{1}(k)}{3}-S_{0}(k)-\frac{S_{\mathrm{T}}(k)}{3} \geq 0 \\
& S_{\mathrm{c} 3}(k)=\frac{1}{2 N k^{2}}\left\langle\left|\sum_{i=1}^{N}\left(\sigma_{i} \times \mathbf{k}\right) \exp \left[i \mathbf{k} \cdot \mathbf{r}_{i}\right]\right|^{2}\right\rangle=1+\frac{S_{1}(k)}{3}-S_{0}(k)+\frac{S_{\mathrm{T}}(k)}{6} \geq 0
\end{aligned}
$$

with

$$
\begin{aligned}
& S_{s}(k)=4 \pi \rho \int_{0}^{\infty}\left[F_{s}(r)-F_{s}(\infty)\right] j_{0}(k r) r^{2} d r, \\
& S_{\mathrm{T}}(k)=4 \pi \rho \int_{0}^{\infty} F_{\mathrm{T}}(r) j_{2}(k r) r^{2} d r .
\end{aligned}
$$

$F_{\mathrm{F} s}(r)$ and $S_{\mathrm{F}}(k)$ in Eq. (2.1) are $F_{S}(r)$ and $S_{\mathrm{c} n}(k)$ in the case of the degenerate Fermi gas, respectively. The last term on the right-hand side of Eq. (2.1), $E_{\mathrm{o}} / N$, is also expressed with $F_{s}(r)$ and $F_{\mathrm{T}}(r)$ explicitly, although we do not show it here because it is a rather lengthy expression [5].

In Eq. (2.1), $E_{2} / N$ is expressed as an explicit functional of $F_{s}(r)$ and $F_{\mathrm{T}}(r)$ (more practically, $F_{\mathrm{C} s}(r)$ and $g_{\mathrm{T}}(r)$ ): Especially, the potential energy is expressed exactly, whereas the kinetic energy is expressed approximately in this EEF. When we assume the Jastrow wave function

$$
\Psi=\operatorname{Sym}\left[\prod_{i>j} f_{i j}\right] \Phi,
$$

with $\Phi$ being the Fermi-gas wave function, we can cluster-expand the expectation value of the Hamiltonian. Then, we confirm that this energy expression appropriately includes the lowest-order two-body-cluster terms, the three-body cluster terms that are the lowest order in the correlations $\left(f_{i j}-1\right)$, and a part of the higher-order cluster terms. Furthermore, EEF automatically guarantees the non-negativeness of $S_{\mathrm{c} n}(k)(n=1,2,3)$ shown in Eqs. (2.4) (2.6).

In this section, we employ the state-independent repulsive part of the UIX potential, $V\left(\mathbf{r}_{1}, \mathbf{r}_{2}, \mathbf{r}_{3}\right)$, as the three-body nuclear force, and then, the three-body energy $E_{3} / N$ is

$$
\frac{E_{3}}{N}=\frac{\rho^{2}}{6} \int F\left(\mathbf{r}_{1}, \mathbf{r}_{2}, \mathbf{r}_{3}\right) V\left(\mathbf{r}_{1}, \mathbf{r}_{2}, \mathbf{r}_{3}\right) d \mathbf{r}_{12} d \mathbf{r}_{23}
$$

Here, we employ an extended Kirkwood's assumption for the three-body distribution function $F\left(\mathbf{r}_{1}, \mathbf{r}_{2}, \mathbf{r}_{3}\right)$ as follows:

$$
F\left(\mathbf{r}_{1}, \mathbf{r}_{2}, \mathbf{r}_{3}\right)=F\left(r_{12}\right) F\left(r_{23}\right) F\left(r_{31}\right) \frac{F_{\mathrm{F}}\left(\mathbf{r}_{1}, \mathbf{r}_{2}, \mathbf{r}_{3}\right)}{F_{\mathrm{F}}\left(r_{12}\right) F_{\mathrm{F}}\left(r_{23}\right) F_{\mathrm{F}}\left(r_{31}\right)},
$$

where $F(r)=F_{1}(r)+F_{0}(r)$, and the functions with the subscript $\mathrm{F}$ are those in the case of the Fermi gas. Then, $E / N=E_{2} / N+E_{3} / N$ is minimized with respect to $F_{\mathrm{Cs}}(r)$ and $g_{\mathrm{T}}(r)$ by solving the Euler-Lagrange equations for them. 

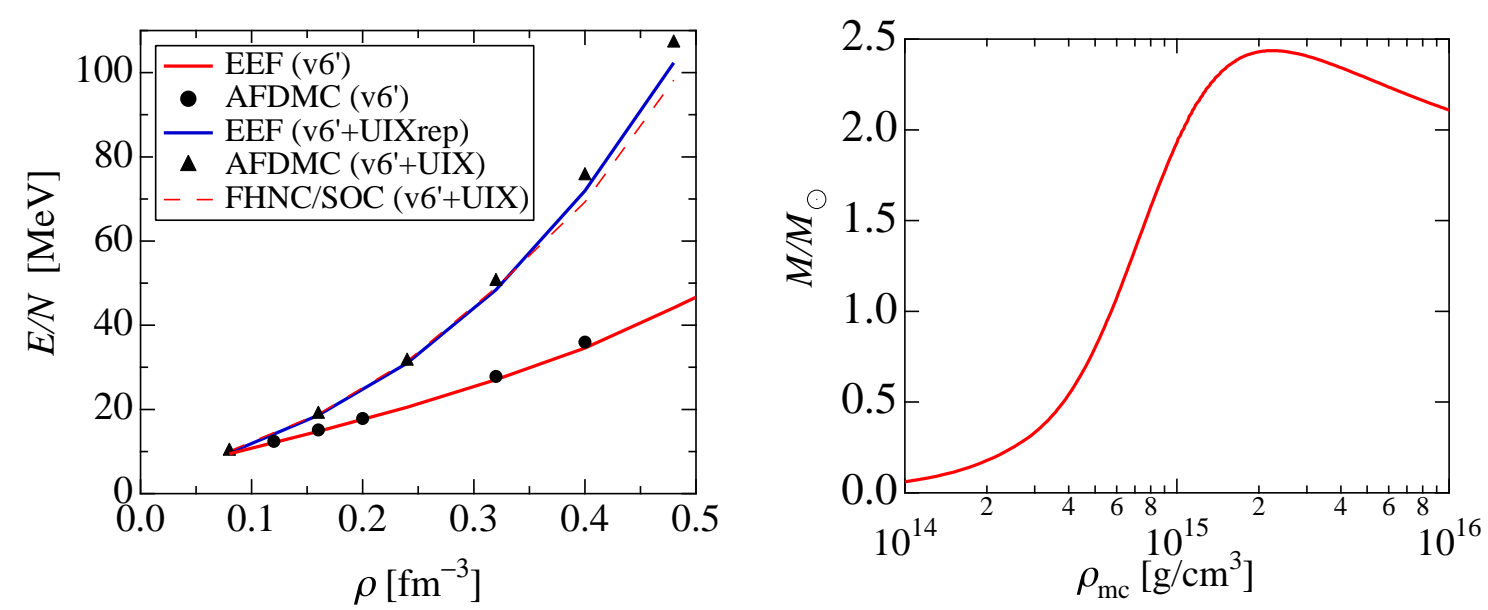

Figure 1: Left: Energy per neutron $E / N$ of neutron matter with the v6' two-body potential and the repulsive part of the UIX three-body potential calculated by EEF. Also shown are those obtained by AFDMC and FHNC with the full UIX potential [焑], as well as $E_{2} / N$ with the v6' potential obtained by EEF and AFDMC [D]. Right: Neutron star masses as a function of the central mass density $\rho_{\mathrm{mc}}$ with the present EOS of pure neutron matter.

The left panel in Fig. 1 shows $E / N$ for neutron matter with the v6' two-body potential and the repulsive part of the UIX three-body potential. Also shown are the results obtained by the Auxiliary Field Diffusion Monte Calro (AFDMC) and the Fermi Hypernetted Chain (FHNC) methods [ [6]. It is seen that $E / N$ with the EEF is close to those obtained by AFDMC and FHNC. It should be noted that, in the latter two cases, the $2 \pi$-exchange part of the UIX potential is also taken into account. The closeness of our results to those obtained by AFDMC and FHNC may imply that the repulsive part of the UIX predominates over the $2 \pi$-exchange part in normal neutron matter. In this figure, in addition, we compare $E_{2} / N$ without TBF calculated by the EEF and AFDMC [ close to each other. Further detail on this is given in Ref. [[]], including in the case of symmetric nuclear matter. Since the numerical calculation of $E / N$ by the EEF is much easier than the Monte Carlo calculation, we can calculate $E / N$ at densities higher than those given by AFDMC. In fact, with use of our EOS of pure neutron matter, we can see the maximum mass of the neutron star to be about $2.5 M_{\odot}$, as shown in the right panel of Fig. 1.

As the next step of the extension of EEF, we plan to take into account the spin-orbit components of the two-body nuclear potential.

\section{Nuclear EOS for Supernova Simulations based on the Cluster Variational Method}

As an application of the cluster variational method (CVM) to astrophysical phenomena, we are now constructing a new nuclear EOS for SN numerical simulations based on CVM. Since the SNEOS must cover an extremely wide range of baryon density $n_{\mathrm{B}}$, temperature $T$ and proton fraction $Y_{\mathrm{p}}$, we have to treat non-uniform matter as well as uniform nuclear matter. In this project, therefore, we calculate the free energy for uniform matter with CVM at finite temperatures proposed by Schmidt and Pandharipande (SP) [8] , and we treat the non-uniform matter in the Thomas-Fermi 
(TF) approximation by following a route similar to that of Shen et al. [Q] ]. In this section, we report the current status of this project.

For uniform nuclear matter, we start from the nuclear Hamiltonian, which consists of the AV18 two-body potential including the central, tensor, spin-orbit and quadratic-angular-momentum terms, and the UIX three-body nuclear potential. Then, the expectation value of the two-body Hamiltonian $\mathrm{H}_{2}$ with the Jastrow wave function is evaluated in the two-body cluster approximation. Here, we impose the extended Mayer's condition and healing-distance condition, so that the obtained two-body energy per nucleon, $E_{2} / N$, is close to that obtained with the FHNC calculation by Akmal et al. [U]] (APR). Furthermore, we take into account the three-body energy $E_{3} / N$ by modifying the expectation value of the three-body Hamiltonian with the Fermi-gas wave function so that the total energy $E / N=E_{2} / N+E_{3} / N$ reproduces the empirical saturation data with high precision [3], 团].

At finite temperatures, we follow the method of SP for calculating the free energy per nucleon, $F / N$. In this method, $F / N$ is expressed with averaged occupation probabilities of the singleparticle states of nucleons, and those probabilities are parameterized by the effective masses $m_{i}^{*}(i=$ $\mathrm{p}, \mathrm{n})$ of proton and neutron, respectively. Then, $F / N$ is minimized with respect to $m_{i}^{*}$. The obtained $F / N$ is close to the FHNC result that is an extension of $E / N$ by APR to finite temperatures [W]]. It is noted that the FHNC is applicable only to neutron matter and symmetric nuclear matter, whereas the present CVM is applicable to nuclear matter for any proton fractions. Therefore, the present method is suitable for construction of the SN-EOS.

For non-uniform nuclear matter, we employ the TF approximation used by Shen et al. [Q] . In the Wigner-Seitz (WS) approximation, we express the free energy of a WS cell as follows:

$$
F=\int_{\text {cell }} f\left(n_{\mathrm{p}}(r), n_{\mathrm{n}}(r)\right) d \mathbf{r}+F_{0} \int_{\text {cell }}|\nabla n(r)|^{2} d \mathbf{r}+\frac{e^{2}}{2} \iint_{\text {cell }} \frac{\left[n_{\mathrm{p}}(r)-n_{\mathrm{e}}\right]\left[n_{\mathrm{p}}\left(r^{\prime}\right)-n_{\mathrm{e}}\right]}{\left|\mathbf{r}-\mathbf{r}^{\prime}\right|} d \mathbf{r} d \mathbf{r}^{\prime}+\Delta E_{\mathrm{c}}
$$

Here, $f$ is the free energy density of uniform matter calculated with $F / N$ obtained above. The number densities of protons and neutrons $n_{i}(r)(i=\mathrm{p}, \mathrm{n})$ are parameterized as

$$
n_{i}(r)=\left\{\begin{array}{cl}
\left(n_{i}^{\text {in }}-n_{i}^{\text {out }}\right)\left[1-\left(r / R_{i}\right)^{t_{i}}\right]^{3}+n_{i}^{\text {out }} & \left(r \leq R_{i}\right) \\
n_{i}^{\text {out }} & \left(R_{i} \leq r \leq R_{\text {cell }}\right) .
\end{array}\right.
$$

In Eq. (3.1), $n(r)=n_{\mathrm{p}}(r)+n_{\mathrm{n}}(r)$, and $F_{0}$ is chosen to be $68.00 \mathrm{MeVfm}^{5}$ so that the TF calculation reproduces gross features (masses and radii) of isolated atomic nuclei [四]. Electrons are assumed to be distributed uniformly in the WS cell, with the number density being $n_{\mathrm{e}}$. The last term $\Delta E_{\mathrm{c}}$ in Eq. (3.1) is the correction to the Coulomb energy due to the bcc lattice structure. Then, at a given set of $n_{\mathrm{B}}, Y_{\mathrm{p}}$ and $T$, the averaged free energy density in the WS cell is minimized with respect to the parameters in Eq. (3.2).

By using the TF calculation mentioned above, we constructed the phase diagram of nuclear matter at zero temperature, as shown in Fig. 2. It is seen that, as $n_{\mathrm{B}}$ decreases, non-uniform matter distribution becomes favorable: The critical density between uniform and non-uniform phases is about $10^{14.23} \mathrm{~g} / \mathrm{cm}^{3}$, which is slightly higher than in the case of the Shen-EOS $\left(10^{14.2} \mathrm{~g} / \mathrm{cm}^{3}\right)$. At low densities with $Y_{\mathrm{p}} \gtrsim 0.3$, nucleons are bound together to be atomic nuclei. As $Y_{\mathrm{p}}$ decreases, the number of neutrons becomes much larger than that of protons, and then neutrons drip out of the nuclei; in this phase, the nuclei and dripped neutrons coexist. 


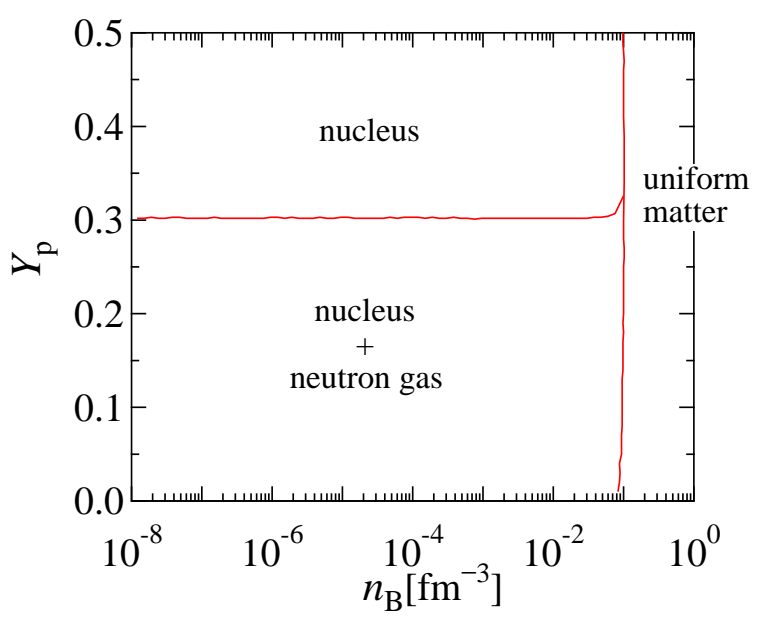

Figure 2: Phase diagram by the present EOS at zero temperature.

We are currently calculating the free energy of non-uniform nuclear matter at finite temperatures with the aim of creating a complete SN-EOS table. It is also important to treat light elements for realistic SN-EOS. As the first step, we are going to include $\alpha$ particles in our EOS, following the method of Shen et al. [Q] .

\section{Acknowledgments}

This work is supported by the Grants-in-Aid for Scientific Research on Innovative Areas of MEXT (Nos. 20105004, 20105005 and 21105510) and by JSTS (No. 21540280).

\section{References}

[1] M. Takano and M. Yamada, Prog. Theor. Phys. 91 (1994) 1149.

[2] M. Takano and M. Yamada, Prog. Theor. Phys. 100 (1998) 745.

[3] H. Kanzawa, K. Oyamatsu, K. Sumiyoshi and M. Takano, Nucl. Phys. A 791 (2007) 232.

[4] H. Kanzawa, M. Takano, K. Sumiyoshi and K. Oyamatsu, Prog. Theor. Phys. 122 (2009) 673.

[5] M. Takano et al., in preparation.

[6] A. Lovato, O. Benhar, S. Fantoni, A. Y. Illarionov and K. E. Schmidt, Phys. Rev. C 83 (2011) 054003.

[7] A. Sarsa, S. Fantoni, K. E. Schmidt and F. Pederiva, Phys. Rev. C 68 (2003) 024308.

[8] K. Schmidt and V. A. Pandharipande, Phys. Lett. B 87 (1979) 11.

[9] H. Shen, H. Toki, K. Oyamatsu and K. Sumiyoshi, Nucl. Phys. A 637 (1998) 435.

H. Shen, H. Toki, K. Oyamatsu and K. Sumiyoshi, Prog. Theor. Phys. 100 (1998) 1013.

[10] A. Akmal, V. A. Pandharipande and D. G. Ravenhall Phys. Rev. C 58 (1998) 1804.

[11] A. Mukherjee, Phys. Rev. C 79 (2009) 045811. 